\title{
THE ASSEMBLY OF CD1e IS CONTROLLED BY AN N-TERMINAL PROPEPTIDE WHICH IS PROCESSED IN ENDOSOMAL COMPARTMENTS
}

Blandine Maître ${ }^{*}$, Catherine Angénieux ${ }^{*}$, Virginie $\underline{\text { Wurtz }}^{\dagger}$, Emilie Layre ${ }^{\ddagger}$, Martine Gilleron ${ }^{*}$, Anthony Collmann $^{\S}$, Sabrina Mariotti ${ }^{\S}$, Lucia $\underline{\text { Mori }}^{\S}$, Dominique Fricker ${ }^{*}$, Jean-Pierre

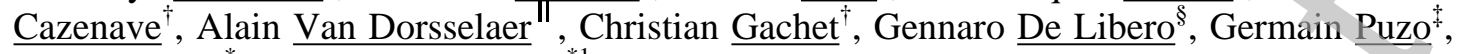
Daniel $\underline{\text { Hanau }}^{*}$ and Henri de la Salle ${ }^{* 1}$

INSERM, "U.725 "Biology of Human Dendritic Cells" and "U.311 "Biologie et pharmacologie de l'hémostase et de la thrombose", Strasbourg, F-67065, France ; Etablissement Français du Sang-Alsace, Strasbourg, F-67065, France ; Université LouisPasteur, Strasbourg F-67000, France. ${ }^{*}$ CNRS UMR 5089, "Immunochimie et Glycoconjugués Mycobactériens", Institut de Pharmacologie et de Biologie Structurale, Toulouse, F-31077, France. ${ }^{\S}$ Experimental Immunolggy, Department of Biomedicine, University Hospital Basel, CH-4031, Basel, Switzerland. 'UMR 7178 (CNRS-ULP), Ecole Européenne de Chimie Polymères et Matériaux, Laboratoire de Spectrométrie de Masse Bio-Organique, Institut Pluridisciplinaire Hubert Curien, Strasbourg, F-67087, France.

${ }^{1}$ To whom correspondence should be addressed: H. de la Salle, INSERM U.725; Etablissement Français du Sang-Alsace ; 10 rue Spielmann, 67065 Strasbourg, France. Tel.: 333882125 25; Fax: 333882125 44; E-mail: henri.delasalle@efs-alsace.fr

Short title: Double endosomal processing of CD1e

Key words : CD1, PIM, propeptide, antigen presentation

Abbreviations used : immature, iDCs, and mature, mDCs, dendritic cells; membraneassociated, mCD1e, and soluble CDle, sCD1e; phosphatidylinositol hexa- or di-mannoside, $\mathrm{PIM}_{6}$ or $\mathrm{PIM}_{2}$; recombinant soluble, rs; endoglycosidase, Endo H ; Peptide: N-glycosidase F, PNGase F. 


\section{Summary}

CD1e displays unique features in comparison to other CD1 proteins. CD1e accumulates in Golgi compartments of immature dendritic cells and is directly transported to lysosomes, where it is cleaved in a soluble form. In these latter compartments, CD1e participates in the processing of glycolipid antigens. Here we show that the $\mathrm{N}$-terminal end of the membrane-associated molecule begins at aminoacid 20, while the soluble molecule consists of aminoacids 32 to 333 . Thus, immature CD1e includes an N-terminal propeptide which is cleaved in acidic compartment and so, absent on the mature endosomal form. Mutagenesis experiments demonstrated that the propeptide controls the assembly of the CD1e $\alpha$ chain with $\beta 2$-microglobulin while propeptide-deleted CD1e molecules are immunologically active. Comparison of CD1e cDNÁs from different mammalian species indicates that the CD1e propeptide is conserved during evolution, suggesting that it may also optimize the generation of CD1e molecules in other species. 


\section{Introduction}

CD1 molecules are non classical HLA class I molecules, among which human CD1e displays several distinct features in its trafficking, function and primary structure. Human CD1a, b, c and $\mathrm{d}$ cycle between the plasma membrane and different endosomal compartments, and are involved in the presentation of glycolipids to $\mathrm{T}$ cells [1]. Conversely, CD1e initially accumulates in the Golgi compartments of immature dendritic cells (iDCs) or transfected cells as a membrane-associated form (mCD1e) and is subsequently directly transported to early and then late endosomes, where it is cleaved into a soluble form (sCD1e) $[2,3]$. This form facilitates the processing of glycolipids presented by other CD1 molecules transiting through the same compartments. For example, presentation of phosphatidylinositol hexamannoside $\left(\mathrm{PIM}_{6}\right)$ to CD1b-restricted $\mathrm{T}$ cell clones is CD1e-dependent, whereas the presentation of dimannoside $\left(\mathrm{PIM}_{2}\right)$ is not [4]. In vitro experiments have shown that $\alpha$-mannosidase digests $\mathrm{PIM}_{6}$ into the immunogenic $\mathrm{PIM}_{2}$ form, only in the presence of sCD1e [4].

Alignment of the mature $\alpha$ chains of human CD1 proteins, without the signal peptide, suggests that CD1e has an N-terminal extension of 8-10 aminoacids (Table I, panel A). The assumption that this extension is present on endosomal CD1e is however not supported by the $\mathrm{N}$-terminal aminoacid sequence of a sheep thymic CD1 protein [5] which should correspond to endosomal sCD1e on the basis of its sequence homologies and biochemical properties. This latter sequence suggests that the $12 \mathrm{~N}$-terminal aminoacids of buman CD1e are cleaved. As the gene sequence of sheep CD1e is not known, it is not possible to confirm N-terminus processing of CD1e in this animal.

Comparison of CD1e proteins in different species suggests the existence of an $\mathrm{N}$-terminal extension conserved during evolution (Table I, panel B), which is cleaved during CD1e maturation. The aim of the present study was to validate this hypothesis, to identify the cellular compartments where this $\mathrm{N}$-terminal extension is cleaved and to elucidate its function.

\section{Experimental}

\section{Genetic constructions}

CD1e forms used in this work were derived from the cDNA encoding the isoform with the long cytoplasmic tail $(\mathrm{CD} 1 \mathrm{eL},[2])$. Propeptide-deleted molecules were derived by PCR mutagenesis of cDNAs encoding CD1e. Various CD1e-derived molecules were expressed in the pEGFP-N3 vector (Clontech-Takara Bio Europe, Saint Germain en Laye, France)

\section{Antibodies.}

The anti-CD1e mAbs VIIC7, 2.9 and 20.6 have been described previously [2]. The anti- $\beta 2 \mathrm{~m}$ mAb B1G6 was purchased from Beckman-Coulter (Villepinte, France). Polyclonal Abs specific for the $\mathrm{N}$-terminus of neosynthesized CD1e were raised by immunizing rabbits with the synthetic peptide APQALQSYHLAAEEC (NeoMPS, Strasbourg, France) coupled to keyhole limpet hemocyanin. Anti-peptide Abs were immunopurified from $5 \mathrm{ml}$ of antiserum by adsorption onto $1 \mathrm{mg}$ of peptide coupled to NHS-activated Sepharose (GE Healthcare, Orsay, France). CD1e molecules were immunopurified with anti-CD1e mAbs (clone 20.6) chemically coupled with dimethyl pimelimidate (Perbio Science, Brebière, France) on protein A Sepharose (GE Healthcare).

\section{Transfected cells and biochemical analyses}

Transfected M10 cells expressing CD1e and CD1b molecules, alone or together, have been previously described [2, 4]. Mutated forms of CD1e were expressed in M10 cells, expressing or not CD1b, after transfection with Fugene 6 reagent (Roche Applied Science) followed by 
selection in the presence of $500 \mu \mathrm{g} / \mathrm{ml} \mathrm{G} 418$ (Invitrogen, Cergy Pontoise, France). Clones expressing CD1e were identified by immunofluorescence of fixed permeabilized cells stained with the mAb 20.6, respectively, as described [2].

In western blotting analyses, cells were recovered from a $75 \mathrm{~cm}^{2}$ flask, resuspended in lysis buffer $\left(150 \mathrm{mM} \mathrm{NaCl}, 20 \mathrm{mM}\right.$ Tris $\mathrm{pH} 7.5$, containing $1 \%$ Triton X100 and Complete ${ }^{\mathrm{TM}}$ protease inhibitor cocktail, Roche Applied Science) and centrifuged at $6000 \mathrm{~g}$ to eliminate the nuclei. CD1e molecules were immunoprecipitated for $2 \mathrm{~h}$ using $50 \mu 1$ of either VIIC7 or 20.6 hybridoma cell culture supernatant and adsorbed onto protein A Sepharose. After extensive washing, proteins were eluted from the matrix with $0.5 \%$ SDS and treated or not with endoglycosidase (Endo) H or Peptide: N-glycosidase F (PNGase F) (New England Biolabs, Beverly, MA). The proteins were separated by SDS-PAGE, transferred to nitrocellulose membranes and incubated with the mAb VIIC7 or anti-A15C polyclonal Abs, followed by HRP-conjugated goat anti-mouse or anti-rabbit Abs, respectively. Bands were revealed with SuperSignal West Pico reagent (Perbio Science). Pulse-chase metabolic labeling, immunoprecipitations and Endo $\mathrm{H}$ or $\mathrm{F}$ treatment were carried out as previously described [2]. Molecular mass standards used were either prestained protein markers (New England Biolabs) $(47.5,32.5$ and $25 \mathrm{kD})$ or $14 \mathrm{C}$-labeled GE-Healthcare (45 and $30 \mathrm{kD})$.

\section{Purification of CDle molecules from transfected cells}

Membrane and cytoplasmic proteins from $7 \times 10^{8}$ stably transfected cells were extracted in 30 $\mathrm{ml}$ of lysis buffer (Tris $20 \mathrm{mM}, \mathrm{NaCl} 150 \mathrm{mM}, 1 \%$ Triton X100 and protease inhibitors, $\mathrm{pH}$ 7.5), pre-immunoadsorbed on protein A Sepharose overnight and then incubated for $2 \mathrm{~h}$ with $40 \mu \mathrm{g}$ of 20.6 covalently coupled to protein A Sepharose. After extensive washing, the immunoadsorbed proteins were eluted, separated by SDS-PAGE and revealed with Coomassie blue G250.

Mass spectrometry of tryptic digests

Immunopurified recombinant CD1e molecules were separated on SDS-PAGE and stained with Coomassie Blue. As a negative control, identical procedure was performed using untransfected cells. Major species specific for transfected cell extracts, corresponding to mCD1e and sCD1e, were cut. Gel slices were washed twice with $50 \mu \mathrm{l}$ of $25 \mathrm{mM} \mathrm{NH}_{4} \mathrm{HCO}_{3}$ followed by $50 \mu 1$ of acetonitrile. Cysteine residues were reduced by $50 \mu 1$ of $10 \mathrm{mM}$ dithiothreitol at $57^{\circ} \mathrm{C}$ and alkylated by $50 \mu 1$ of $55 \mathrm{mM}$ iodoacetamide. After dehydratation with acetonitrile, the proteins were cleaved in gel with a $12.5 \mathrm{ng} / \mu \mathrm{l}$ solution of modified trypsin (Promega, Madison, WI, USA) in $25 \mathrm{mM} \mathrm{NH}_{4} \mathrm{HCO}_{3}$. The resulting tryptic peptides were extracted with $60 \%$ acetonitrile in $5 \%$ formic acid and analyzed by LC-MS/MS.

Measurements by MALDI mass spectrometry were carried out on an Ultraflex TOF/TOF (Bruker, Germany) mass spectrometer in reflectron mode with positive ion spectra. The samples were prepared by standard dried droplet preparation on stainless steel MALDI targets using $\alpha$-cyano-4-hydroxycinnamic acid as matrix. External calibration of MALDI mass spectra was carried using singly monoisotopic peaks of a mixture of bradykinin 1-7 $(\mathrm{m} / \mathrm{z}=757.400)$, human angiotensin II $(\mathrm{m} / \mathrm{z}=1046.542)$, human angiotensin $\mathrm{I}(\mathrm{m} / \mathrm{z}=1296.685)$, substance $\mathrm{P}(\mathrm{m} / \mathrm{z}=1347.735)$, bombesin $(\mathrm{m} / \mathrm{z}=1619.822)$, rennin $(\mathrm{m} / \mathrm{z}=1758.933)$, ACTH 1-17 $(\mathrm{m} / \mathrm{z}=2093.087)$ and ACTH 18-39 $(\mathrm{m} / \mathrm{z}=2465.199)$. To achieve mass accuracy, internal calibration was performed with trypic peptides coming from autolysis of trypsin, with monoisotopic masses automatically annoted using Flexanalysis 2.0.

For nano-HPLC, a CapLC system (Micromass Ltd., Manchester, UK) was used. The samples were concentrated on a precolumn, and the peptides were separated on a $15 \mathrm{~cm} \times 75 \mu \mathrm{m}$ internal diameter column packed with $3 \mu \mathrm{m} 100 \AA$ A 18 PepMap (LC-Packings). The MS and 
MS/MS analyses were performed with a Q-TOF 2 hybrid quadrupole/time-of-flight mass spectrometer (Micromass Ltd., Manchester, UK) equipped with a Z-spray ion source.

Mass data collected during LC-MS/MS analysis was processed and converted into a PKL file to be submitted to the search software Mascot (Matrix Science Ltd., London, UK). Searches were performed with a tolerance on mass measurement of $0.25 \mathrm{Da}$ in MS and MS/MS mode in NCBI (The National Center for Biotechnology Information) database.

Production of recombinant soluble ( $r s)$ CDle in insect cells

The luminal domains of CD1e without the signal peptide, beginning at Ala 20 (rsCD1e) or Glu 32 (rs $\Delta 12 \mathrm{CD} 1 \mathrm{e}$ ) and ending at Ser 305, were directly fused to the signal peptide of BIP and a C-terminal V5His tag (pMTbipV5HisA, Invitrogen). The two forms were expressed in S2 Drosphila cells and purified from culture medium as previously described $[4,6]$. The resulting preparations included only the CD1e $\alpha$ chain and $\beta 2-\mathrm{m}$, as shown by SDS-PAGE analysis. N-terminal aminoacid sequencing confirmed that rsCD1e and rs $\Delta 12 \mathrm{CD} 1 \mathrm{e}$ began with the expected aminoacid sequence APQALQ or EEQLSF, respectively (data not shown).

\section{Antigen presentation assays}

Transfected M10 cells (3×10\% $/$ well) growing in RPMI-1640 medium supplemented with $10 \%$ FCS, $10 \mathrm{mM}$ HEPES, $2 \mathrm{mM}$ Ultraglutamine II, MEM nonessential amino acids, $1 \mathrm{mM}$ sodium pyruvate (all from Lonza, Brussels, Belgium) and $100 \mu \mathrm{g} / \mathrm{ml}$ kanamycin (Invitrogen, Basel, Switzerland) were preincubated for $2 \mathrm{~h}$ at $37^{\circ} \mathrm{C}$ with different concentrations of purified $\mathrm{PIM}_{6}[7,8]$, or GM1 ganglioside (Matreya, Pleasant Gap, PA), before addition of T cells $\left(10 \%\right.$ well in triplicates). The T cells used were the CD1b-restricted, PIM $_{6}$-specific and CD1e-dependent clone DL15A30, and the CD1b-restricted and GM1-specific clone GG33A [9], which is CD1e-independent [4]. Supernatants were harvested after $36 \mathrm{~h}$ of incubation, and cytokine release was measured with an enzyme-linked immunosorbent assay for GM-CSF or IFN- $\gamma$ (ELISA) kit (R\&D Systems, Abingdon, UK). Data were expressed as mean arbitrary units (A.U. for GM-CSF) or $\mathrm{pg} / \mathrm{ml}$ (for IFN- $\gamma$ ) \pm the standard deviation (SD) of triplicates.

The biological activity of rsCD1e was also tested by pre-incubating overnight CD1btransfected M10 cells with $20 \mu \mathrm{g} / \mathrm{ml}$ rsCD1e. Cells were then washed and processed for antigen presentation as described above.

Enzymatic digestion of PIM6.

Purified $\mathrm{PIM}_{6}(100 \mu \mathrm{g}$ ) was sonicated in $50 \mu 1$ of mannosidase buffer (sodium acetate $0.1 \mathrm{M}$, $\mathrm{ZnSO}_{4} 1 \mathrm{mM} \mathrm{pH} 4.5$ ). RsCD1e (4.3 nanomoles in $150 \mu \mathrm{l}$ ) was equilibrated by dialysis against mannosidase buffer for $1 \mathrm{~h}$ and then incubated with $\mathrm{PIM}_{6}(100 \mu \mathrm{g}$ in $50 \mu \mathrm{l})$ and with jack bean a-mannosidase $\left(20 \mu \mathrm{l}, 1.8 \mathrm{mg} / \mathrm{ml}\right.$, Sigma) for $1 \mathrm{~h}$ at $37{ }^{\circ} \mathrm{C}$. Glycolipids were extracted and analyzed by mass spectrometry as previously described [4].

\section{Results}

Characterization of the ends of CDle forms provides evidence for processing of the $N$ terminus

Analysis of pre-CD1e sequence predicts that cleavage of the signal peptide occurs between Ala19 and Ala20 (Table IA), whereas data from a CD1e-like sheep protein indicate that the 12 following N-terminal aminoacids have been removed from the lysosomal form. To confirm these predictions, CD1e molecules from transfected M10 cells were immunopurified and separated by SDS-PAGE. After Coomassie blue staining, two protein species were recovered having apparent molecular masses corresponding to those of mCD1e and sCD1e (Fig. 1A). Comparison of the MALDI-TOF mass spectra of the tryptic digests of the two forms of CD1e revealed four peaks of $\mathrm{m} / \mathrm{z} 1050.54,1300.69,1790.89$ and $2159.08 \mathrm{Da}$, 
specific to the $45 \mathrm{kD}$ mCD1e form (Fig. 1B). These peaks were not predicted by in silico trypsin digestion of mCD1e and hence probably corresponded to semi-tryptic species (with only one trypsin-generated end) resulting from digestion of the $\mathrm{N}$ - and $\mathrm{C}$ - terminal ends of $\mathrm{mCD} 1 \mathrm{e}$. On the basis of this hypothesis, the $\mathrm{m} / \mathrm{z}$ value of the predominant $2159.08 \mathrm{Da}$ peak corresponded to the theoretical $\mathrm{m} / \mathrm{z}$ value of the peptide APQALQSYHLAAEEQLSFR, generated by trypsin digestion of the predicted N-terminal end of mCD1e (Table IA). The three other peaks represented shorter N-terminal ends (Table II). The identities of these peptides were confirmed by de novo sequencing after LC-MS/MS (Fig. 1C).

A similar analysis of the tryptic digest of $\mathrm{sCD} 1 \mathrm{e}$ revealed three prominent peaks of $\mathrm{m} / \mathrm{z}$ 908.45, m/z 1585.80 and m/z 1913.00 Da (Fig. 1B). LC-MS/MS showed that the 1913.00 Da peak corresponded to the internal tryptic peptide NLQSLFQLYFHSFIR (theoretical molecular mass 1912.00), which represents aminoacids 92-106 of preCD1e (data not shown). Since a glycosylation site is predicted at Asn 84, it is possible that the processing of carbohydrates in lysosomes results in a greater accessibility to trypsin of the aminoester bond between aminoacids 91 and 92, which would explain why this peak was only observed in the tryptic digest of sCD1e. The two first peaks were not predicted by in silico trypsin digestion of CD1e and thus might result from digestion of the $\mathrm{N}$ - or C- terminal end of sCD1e. This conclusion was confirmed by de novo sequencing after LC-MS/MS (Fig.1C, Table II). The $\mathrm{m} / \mathrm{z}$ 908.45 Da species corresponded to a peptide derived by proteolysis of the $\mathrm{N}$-terminal end of sCD1e, EEQLSFR, resulting from the cleavage of the $12 \mathrm{~N}$-terminal aminoacidsof mCD1e. The aminoacid sequence of the $\mathrm{m} / \mathrm{z} 1585.80$ Da entity was HSSLGGHDLIIHWGG (theoretical $\mathrm{m} / \mathrm{z} 1585.78 \mathrm{Da}$ ) and hence corresponded to the C-terminal end of sCD1e. This determination confirmed that $\mathrm{SCD} 1 \mathrm{e}$ is generated by a proteolytic cleavage of the luminal part of mCD1e, near the transmembrane domain.

Thus, newly synthesized CD1e molecules include a 12-aminoacid N-terminal extension, hereafter called the propeptide, which is absent from lysosomal sCD1e. Subsequent experiments were designed to identify the cellular compartment where the propeptide is cleaved.

\section{The $12 \mathrm{~N}$-terminal aminoacids of CDle are cleaved in endosomes}

To confirm that these 12 aminoacids are present on ER-localized CD1e molecules, polyclonal antibodies against a synthetic peptide including the $12 \mathrm{~N}$-terminal aminoacids of $\mathrm{CD} 1 \mathrm{e}$ (A15C) were raised in rabbits. CD1e was immunoprecipitated from extracts of transfected M10 cells expressing CD1e using the mAb VIIC7. This mAb recognizes the cytoplasmic domain of $\mathrm{CD} 1 \mathrm{e}$ and in transfected M10 cells immunoprecipitates molecules which are EndoH sensitive and thus located in the ER [2]. The immunoadsorbed CD1e was then analyzed by western blotting using either VIIC7 or the polyclonal anti-A15C Abs (Fig. 2). As compared to VIIC7 mAb, A15C Ab poorly detected CD1e molecules, which may explain why it only revealed EndoH-sensitive CD1e molecules immunoprecipitated with the mAb VIIC7. Nevertheless, these experiments demonstrated that the anti-propeptide Abs recognized EndoH sensitive, ER-localized, CD1e molecules.

To confirm that the N-terminal extension is missing on lysosomal sCD1e and to identify the cellular compartment in which it is processed, a CD1e form deleted of its $12 \mathrm{~N}$-terminal aminoacids $(\triangle 12 \mathrm{CD} 1 \mathrm{e})$ was expressed in M10 cells. Confocal immunofluorescence microscopy showed that $\mathrm{CD} 1 \mathrm{e}$ and $\Delta 12 \mathrm{CD} 1 \mathrm{e}$ have a similar cellular distribution, accumulating in TGN46 ${ }^{+}$compartments of the Trans Golgi Network and CD63 ${ }^{+}$lysosomal compartments (supplementary data 1). Then, the biochemical maturation of $\Delta 12 \mathrm{CD} 1 \mathrm{e}$ was compared to that of complete $\mathrm{CD} 1 \mathrm{e}$ by pulse-chase labeling experiments, and immunoprecipitation with the $\mathrm{mAb}$ 20.6. This $\mathrm{mAb}$ immunoprecipitates Golgi- and 
endosome-localized CD1e molecules as well as a small percentage of molecules in the ER [2]. Immediately after the pulse and during chase, the mCD1e and $m \Delta 12 C D 1 e$ forms displayed the expected small differences in electrophoretic mobilities (Fig. 3A, bands m). In contrast, after $2 \mathrm{~h}$ of chase, the cleaved molecules (Fig. 3A, bands s) had the same electrophoretic mobility, demonstrating the absence of the $12 \mathrm{~N}$-terminal aminoacids on lysosomal CD1e.

Thus, after 4 hours of chase, the N-terminal extension is still present on mCD1e molecules, which are Endo H-resistant and thus are transiting, or have transited, through the Golgi apparatus [2] (see also Fig. 4). Thus, likely, the propeptide is cleaved in a post-Golgi compartment. To further investigate this mechanism of $\mathrm{CD} 1 \mathrm{e}$ processing, transfected cells expressing CD1e or $\Delta 12 \mathrm{CD} 1 \mathrm{e}$ were pulse labeled and chased for $4 \mathrm{~h}$ in the presence of bafilomycin, after which CD1e was immunoprecipitated with the mAb 20.6, deglycosylated and analyzed by SDS-PAGE (Fig. 3B). The electrophoretic mobilities of mCD1e and $\mathrm{m} \Delta 12 \mathrm{CD} 1 \mathrm{e}$ remained constant throughout the $4 \mathrm{~h}$ of chase in the presence of bafilomycin. Within $4 \mathrm{~h}$ of chase in the absence of bafilomycin, the majority of full length CD1e molecules (i.e. with propeptide) leave the Golgi compartments, reach late endosomes and become fully processed ([2], see also Fig. 4)

Since bafilomycin blocks the endosome acidification and transport from late endosomes to lysosomes [10], but not transport from Golgi compartments to endosomes, the full length $\mathrm{CD} 1 \mathrm{e}$ molecules immunoprecipitated after $4 \mathrm{~h}$ of chase in the presence of bafilomycin were mostly derived from late endosomes. Thus, bafilomycin blocks both the generation of sCD1e, as already reported [2], and cleavage of its $12 \mathrm{~N}$-terminal residues. We previously found that a $27 \mathrm{kD}$ protein co-immunoprecipitates with $\mathrm{CD} 1 \mathrm{e}$ from cell extracts of bafilomycin-treated cells $[2,11]$. Since this protein is PNGase resistant, it is unrelated to CD1e. Metabolic experiments here demonstrate that this interaction is preserved after deletion of the propeptide. These experiments demonstrate that the CD1e propeptide is cleaved by $\mathrm{pH}$ dependent mechanism in late endosomal compartments.

\section{The propeptide controls the exit of CDle from the ER}

Propeptides have very diverse functions. They can be involved, for example, in controlling the ER exit of proteins (as in the case of proalbumin, apolipoprotein A-I or ADAMTS9), in post-translational modifications (vitamin K-dependent blood clotting factors), or in regulating the activity of pro-proteases. To investigate whether maturation of the CD1e protein is controlled by the propeptide, we performed semi-quantitative biochemical studies using pulse-chase labeling followed by immunoprecipitation with different anti-CD1e mAbs. Immunoprecipitation with the mAb VIIC7 (Fig. 4A), which recognizes all mCD1e molecules, even those which are not assembled with $\beta 2 \mathrm{~m}$, revealed no major differences between CD1e and $\triangle 12 \mathrm{CD} 1 \mathrm{e}$ forms. Densitometry scanning showed that during chase the amounts of immunoprecipitated CD1e and $\triangle 12 \mathrm{CD} 1 \mathrm{e}$ decreased with similar kinetics (data not shown).

Interestingly, immunoprecipitation with the conformation-dependent mAb 20.6 gave more informative results since the kinetics of the generation of soluble $\triangle 12 \mathrm{CD} 1 \mathrm{e}$ were significantly slower than for soluble wild type CD1e (Fig. 4A-C). Moreover, the generation of EndoHresistant $\triangle 12 \mathrm{CD} / \mathrm{e}$ molecules, ie molecules reaching the Golgi compartments, appeared to be delayed throughout the 8 hours of chase. In particular, EndoH sensitive $\mathrm{m} \Delta 12 \mathrm{CD} 1 \mathrm{e}$ molecules were still detected at the end of the chase (Fig. 4A, bands m, Endo H-treated samples). Hence $\triangle 12 \mathrm{CD} 1 \mathrm{e}$ molecules exit the ER more slowly from the ER than wild type CD1e molecules.

This last finding suggests that the propeptide might control the assembly of $\beta 2 \mathrm{~m} / \mathrm{CD} 1 \mathrm{e} \alpha$ chain heterodimers, and/or their exit from the ER. To discriminate between these two possibilities, transfected cells expressing complete or propeptide-deleted CD1e molecules 
were pulse-labeled and chased for different periods of time (Fig. 4B). After immunodepletion with the mAb W6/32, CD1e molecules were immunoprecipitated using the mAb 20.6 or B1G6, which recognize the CD1e $\alpha$ chain or $\beta 2 \mathrm{~m}$, respectively. The immunodepletion of HLA class I molecules was efficient since relatively small amounts of these molecules were immunoprecipitated with the mAb B1G6. Nevertheless, the presence of HLA $\alpha$ chain s in 20.6 immunoprecipitates suggests that these species resulted from incomplete immunoadsorption of W6/32-HLA class I immune complexes, despite two additional runs of protein A sepharose adsorption. Glycosylated CD1e and HLA class I $\alpha$ chains migrated at similar positions, but the two species were well resolved after deglycosylation. At all time points, after immunoprecipitation with the $\mathrm{mAb} 20.6$, the ratio of $\mathrm{s} \Delta 12 \mathrm{CD} 1 \mathrm{e}$ to $\mathrm{m} \Delta 12 \mathrm{CD} 1 \mathrm{e}$ molecules was less than for complete CD1e (Fig. $4 \mathrm{C}$ ). In contrast, after anti- $\beta 2 \mathrm{~m}$ immunoprecipitation, the ratios were equivalent for $\mathrm{CD} 1 \mathrm{e}$ and $\Delta 12 \mathrm{CD} 1 \mathrm{e}$ after 2 or $4 \mathrm{~h}$ of chase (Fig. 4C). At $8 \mathrm{~h}$ of chase, an unrelated protein was detected migrating as CD1e $\alpha$ chain and prevented clear interpretation.

Taken together, these data suggest that the absence of the propeptide leads to delayed assembly of CD1e $\alpha$ chain with $\beta 2 \mathrm{~m}$, and consequently retards the generation of soluble CD1e.

\section{Propeptide deleted molecules are active}

CD1e is necessary for the presentation of the mycobacterial PIM $_{6}$ antigen by CD1b to specific $\mathrm{T}$ cells and for the in vitro conversion of $\mathrm{PIM}_{6}$ into $\mathrm{PIM}_{2}$ by $\alpha$-mannosidase [4]. We used this antigen processing and presentation model to investigate whether the propeptide is required for proper CD1e function. M10 cells expressing CD1b alone, or co-expressing CD1b together with $\mathrm{CD} 1 \mathrm{e}$ or $\Delta 12 \mathrm{CD} 1 \mathrm{e}$, were incubated with $\mathrm{PIM}_{6}$ and then used to stimulate CD1b-restricted PIM-specific T cells. The double transfected M10 cell expressing CD1b and either CD1e or $\Delta 12 \mathrm{CD} 1 \mathrm{e}$ presented $\mathrm{PIM}_{6}$ with similar efficiency thus showing that $\Delta 12 \mathrm{CD} 1 \mathrm{e}$ is able to assist processing and presentation of this lipid antigen (Fig. 5A). In control experiments, transfected M10 cells only expressing CD1b did not present PIM $_{6}$, confirming the requirement of CD1e for presentation of this antigen. In addition, the CD1b-restricted presentation of GM1 ganglioside, a CD1e-independent antigen, was not influenced by either form of CD1e (Fig. 5B).

The influence of the propeptide on the CD1e chaperone function was also investigated using two recombinant soluble CD1e molecules. M10 cells expressing CD1b only were pulsed overnight with rsCD1e or $\mathrm{rs} \triangle 12 \mathrm{CD} 1 \mathrm{e}$ in order to promote their internalization, then washed and incubated with $\mathrm{PIM}_{6}$, together with specific T cells. The two forms of rsCD1e molecules were equally active in assisting PIM $_{6}$, presentation, confirming that both forms have similar behavior in assisting $\mathrm{PIM}_{6}$ processing and presentation (Fig. 5C). Finally, we looked at the in vitro digestion of PIM 6 by $\alpha$-mannosidase. PIM $_{6}$ was incubated with $\alpha$-mannosidase together with rsCD1e or rs $\triangle 12 C D 1$ e and digestion products were analyzed by mass spectrometry. Both CD1e molecules facilitated PIM digestion by $\alpha$-mannosidase as shown by appearance of $\mathrm{PIM}_{2}$ (Fig. 5D).

Taken together, these experiments indicate that the absence of the propeptide delays the assembly and maturation of CD1e but does not alter its immunological function.

\section{Discussion}

CD1e is a unique molecule among the CD1 family members. It is the only CD1 protein not expressed on the cell surface, and it is the only protein to be converted into a soluble lysosomal form. Its immunological relevance has recently been identified [4], however, it remains unclear how it matures and becomes immunologically functional. In 
order to better characterize the biochemical maturation of $\mathrm{CD} 1 \mathrm{e}$, we determined the $\mathrm{C}$ terminal sequence of lysosomal sCD1e molecules and compared the N-terminal sequences of the soluble and membrane-associated forms by analyzing their tryptic digests by mass spectrometry. These analyses confirmed that $\mathrm{sCD} 1 \mathrm{e}$ is generated by cleavage of the luminal part of the molecule, proximal to the transmembrane domain. Several peaks of the of tryptic digest of mCD1e and corresponding to the $\mathrm{N}$-terminal sequences were identified. The major one was deduced by signal peptide prediction algorithm, while the shorter ones may result from processing by ER and/or Golgi-localized protease(s) or from proteolytic events during the purification of CD1e. The major finding of our study was that CD1e is synthesized as a precursor bearing an $\mathrm{N}$-terminal propeptide of 12 aminoacids. This propeptide is processed within endosomes and occurs nearly concomitantly with the generation soluble CD1e. In addition, preliminary data suggest that the two processes are independent.

Pulse chase labeling experiments demonstrated that in contrast to wild type CD1e, more Endo $\mathrm{H}$-sensitive $\triangle 12 \mathrm{CD} 1 \mathrm{e}$ molecules were immunoprecipitated by the conformation-dependent $\mathrm{mAb} 20.6$ at all time points. Similar results were obtained when the mAb 2.9 was used in immunoprecipitation experiments (data not shown). In contrast, the kinetics of maturation of $\mathrm{CD} 1 \mathrm{e}$ and $\triangle 12 \mathrm{CD} 1 \mathrm{e}$ molecules immunoprecipitated with an anti- $\beta 2 \mathrm{~m} \mathrm{mAb}$ were comparable. This strongly suggests that the exit of CD1e/ $\beta 2 \mathrm{~m}$ complexes from the ER does not depend on the propeptide and that the latter mainly facilitates their assembly. A requirement for propeptides in the secretion of other proproteins has been described and one important issue is how they influence ER retention. The glycosylation of three asparagines in its 287 aminoacid long propeptide controls secretion ADAMTS9 metalloprotease [12]. Apolipoprotein A-I is synthesized with a 6-aminoacid propeptide and accumulates in Golgi compartments before being secreted. Deletion of the propeptide leads to partial retention of apolipoprotein A-I in the ER and to its almost complete disappearance in other cellular compartments [13]. Secretion of rat proalbumin is also controlled by a propeptide, since its deletion induces ER retention and very poor protein release [14]. How the short propeptides influence the secretion of these proteins has not been elucidated.

While CD1e propeptide facilitates CDle assembly, propeptide-deleted CD1e molecules are immunologically functional. Indeed, cells expressing $\Delta 12 \mathrm{CD} 1 \mathrm{e}$, which lacks the propeptide, presented the CD1e-dependent PIM $_{6}$ antigen with similar efficiency as cells expressing intact CD1e. Furthermore, rs $\Delta 12 \mathrm{CD} 1 \mathrm{e}$ produced in insect cells was able to complement M10 cells only expressing CD1b for the presentation of PIM6, like rsCD1e molecules [4]. Finally, in vitro, the two forms of recombinant soluble CD1e similarly facilitated complete digestion of $\mathrm{PIM}_{6}$ by $\alpha$-mannosidase.

In conclusion, our studies demonstrate that the CD1e propeptide facilitates the assembly of CD1e in the ER, and consequently its egress and transport to Golgi compartments. This is conserved in all species in which the CD1e cDNA has been characterized, thus suggesting that it may optimize the generation of CD1e also in other species. It remains to be elucidated why only CD1e displays such a property among CD1 molecules and other members of the MHC class I family.

Acknowledgments. The authors are grateful to J. Mulvihill for excellent editorial assistance, François Signorino-Gelo for expert technical assistance, and Transgène SA (Strasbourg) for providing access to their adenovirus recombination tools. The authors have no financial conflict of interest.

Funding 
This work was supported by INSERM and EFS-Alsace, the National Research Agency (grant ANR-05-MIME-006), the Swiss National Fund (grant 3100A0-109918) and the EU TB-VAC grant (QLK-CT-1999-01093). BM was the recipient of a grant from ARMESA. 


\section{REFERENCES}

1 Vincent, M. S., Gumperz, J. E. and Brenner, M. B. (2003) Understanding the function of CD1-restricted T cells. Nat. Immunol. 4, 517-523

2 Angenieux, C., Salamero, J., Fricker, D., Cazenave, J. P., Goud, B., Hanau, D. and de La Salle, H. (2000) Characterization of CD1e, a third type of CD1 molecule expressed in dendritic cells. J. Biol. Chem. 275, 37757-37764

3 Angenieux, C., Fraisier, V., Maitre, B., Racine, V., van der Wel, N., Fricker, D., Proamer, F., Sachse, M., Cazenave, J. P., Peters, P., Goud, B., Hanau, D., Sibarita, J. B., Salamero, J. and de la Salle, H. (2005) The Cellular Pathway of CD1e in Immature and Maturing Dendritic Cells. Traffic 6, 286-302

4 de la Salle, H., Mariotti, S., Angenieux, C., Gilleron, M., Garcia-Alles, L. F., Malm, D., Berg, T., Paoletti, S., Maitre, B., Mourey, L., Salamero, J., Cazenave, J. P., Hanau, D., Mori, L., Puzo, G. and De Libero, G. (2005) Assistance of microbial glycolipid antigen processing by CD1e. Science 310, 1321-1324

5 Hopkins, J., Dutia, B. M. and Rhind, S. M. (2000) Sheep CD1 genes and proteins. Vet. Immunol. Immunopathol. 73, 3-14

6 Tourne, S., Maitre, B., Collmann, A., Layre, E., Mariotti, S., Signorino-Gelo, F., Loch, C., Salamero, J., Gilleron, M., Angenieux, C., Cazenave, J. P., Mori, L., Hanau, D., Puzo, G., De Libero, G. and de la Salle, H. (2008) Cutting edge: a naturally occurring mutation in CD1e impairs lipid antigen presentation. J. Immunol. 180, 3642-3646

7 Gilleron, M., Quesniaux, V. F. and Puzo, G. (2003) Acylation state of the phosphatidylinositol hexamannosides from Mycobacterium bovis bacillus Calmette Guerin and mycobacterium tuberculosis $\mathrm{H} 37 \mathrm{Rv}$ and its implication in Toll-like receptor response. J. Biol. Chem. 278, 29880-29889

8 Gilleron, M., Stenger, S., Mazorra, Z., Wittke, F., Mariotti, S., Bohmer, G., Prandi, J., Mori, L., Puzo, G. and De Libero, G. (2004) Diacylated sulfoglycolipids are novel mycobacterial antigens stimulating $\mathrm{CD} 1$-restricted $\mathrm{T}$ cells during infection with Mycobacterium tuberculosis. J. Exp. Med. 199, 649-659

9 Shamshiev, A., Donda, A., Prigozy, T. I., Mori, L., Chigorno, V., Benedict, C. A., Kappos, L., Sonnino, S., Kronenberg, M. and De Libero, G. (2000) The alphabeta T cell response to self-glycolipids shows a novel mechanism of CD1b loading and a requirement for complex oligosaccharides. Immunity 13, 255-264

10 van Weert, A. W., Dunn, K. W., Gueze, H. J., Maxfield, F. R. and Stoorvogel, W. (1995) Transport from late endosomes to lysosomes, but not sorting of integral membrane proteins in endosomes, depends on the vacuolar proton pump. J. Cell. Biol. 130, 821-834

11 Maitre, B., Angenieux, C., Salamero, J., Hanau, D., Fricker, D., Signorino, F., Proamer, F., Cazenave, J. P., Goud, B., Tourne, S. and de la Salle, H. (2008) Control of the intracellular pathway of CD1e. Traffic 9, 431-445

12 Koo, B. H., Longpre, J. M., Somerville, R. P., Alexander, J. P., Leduc, R. and Apte, S. S. (2007) Regulation of ADAMTS9 secretion and enzymatic activity by its propeptide. J. Biol. Chem. 282, 16146-16154

13 McLeod, R. S., Robbins, C., Burns, A., Yao, Z. and Pritchard, P. H. (1994) Deletion of the propeptide of apolipoprotein A-I impairs exit of nascent apolipoprotein A-I from the endoplasmic reticulum. Biochem. J. 302 ( Pt 3), 641-648

14 McCracken, A. A. and Kruse, K. B. (1989) Intracellular transport of rat serum albumin is altered by a genetically engineered deletion of the propeptide. J. Biol. Chem. 264, 2084320846 


\section{Tables and Figures}

FIGURE 1. Characterization of the N- and C-terminal ends of lysosomal sCD1e. CD1e molecules from solubilized extracts of $7 \times 10^{8}$ transfected M10 cells were immunopurified on immobilized 20.6. After separation by SDS-PAGE, proteins were revealed with Coomassie blue $(A)$. Bands corresponding to membrane-associated $(\mathrm{m})$ and soluble (s) CD1e were recovered, digested with trypsin and analyzed by MALDI mass spectrometry. $B$ ). Comparison of the two spectra revealed identities specific to mCD1e $(\Delta)$ and sCD1e $(*)$. C) De novo sequencing after LC-MS/MS of different precursor ions (i) doubly charged $\mathrm{m} / \mathrm{z} 454.7$ (ii) triply charged $\mathrm{m} / \mathrm{z} 529.2$ and (iii) triply charged $\mathrm{m} / \mathrm{z} 720.3$ confirmed the peptide sequences as (i) EEQLSFR attributed to the N-terminus of sCD1e, (ii) HSSLGGHDLIIHWGG attributed to the C-terminus of the sCD1e and (iii) APQALQSYHLAAEEQLSFR attributed to the Nterminus of mCD1e. The $y$ - and $b$ - ion peaks derived from internal fragmentation of peptide bonds provide amino acid sequence information read from the $\mathrm{C}$-terminus and $\mathrm{N}$-terminus of the peptide, respectively.

FIGURE 2. Evidence for an N-terminal extension on CD1e. Untransfected and CD1etransfected M10 cells were lysed and CD1e was immunoprecipitated with the mAb VIIC7. The immunoprecipitated proteins were left untreated $(\mathrm{U})$ or deglycosylated with Endo $\mathrm{H}(\mathrm{H})$ or PNGase F (F) and then analyzed by Western blotting using the mAb VIIC7 or the polyclonal antibody $\mathrm{A} 15 \mathrm{C}$ recognizing the 12-aminoacid $\mathrm{N}$-terminal peptide. Upper panels, transfected cells; lower panels, untransfected cells.

FIGURE 3. The CD1e propeptide is only present on membrane-associated molecules and is cleaved in late endosomal compartments. Transfected M10 cells expressing complete CD1e (C) or the propeptide-deleted CD1e mutant $(\Delta)$ were metabolically labeled with ${ }^{35} \mathrm{~S}$ methionine and cysteine and recovered immediately $A$ ) or chased for 2 or $4 \mathrm{~h}$ in the presence of bafilomycin $(B)$. The cells were then lysed and CD1e was immunoprecipitated with 20.6. Samples were left untreated (U) or deglycosylated with PNGase F (F). Bands $m$ and $s$ correspond to $\mathrm{mCD} 1 \mathrm{e}$ and endosomal $\mathrm{sCD} 1 \mathrm{e}$, respectively. Band $\mathrm{p}$ corresponds to a non $\mathrm{N}$ glycosylated $27 \mathrm{kD}$ protein which co-immunoprecipitates with $\mathrm{CD} 1 \mathrm{e}$ after bafilomycin treatment.

FIGURE 4. The biochemical maturation of CD1e is delayed in the absence of the propeptide. A) Transfected M10 cells expressing CD1e or $\Delta 12 \mathrm{CD} 1 \mathrm{e}$ were pulse labeled and chased for 0 , 2,4 or $8 \mathrm{~h}$. CD1e molecules were recovered from detergent solubilized cell extracts using the mAb VIIC7 or 20.6 and protein A-Sepharose. The immunoadsorbed molecules were left untreated $(\mathrm{U})$ or digested with Endo $\mathrm{H}(\mathrm{H})$ or PNGase F (F) and separated by SDS-PAGE. Gels were exposed for autoradiography for 11 days (VIIC7) or 23 days (20.6). B) Untransfected M10 cells (M10) and transfected cells expressing complete (CD1e) or propeptide-deleted CD1e ( $\triangle 12 \mathrm{CD} 1 \mathrm{e})$ molecules were pulse labeled and then chased for $0,2,4$ or $8 \mathrm{~h}$. After immuno-depletion of HLA class I molecules with the mAb W6/32, CD1e molecules were immunoprecipitated with 20.6 or the anti- $\beta 2 \mathrm{~m} \mathrm{mAb} \mathrm{B1G6}$ then treated with Endo H (H) or PNGase F (F) or not treated (U), separated by SDS-PAGE and revealed by autoradiography. $C$ ) The autoradiograms from two pulse-chase labeling experiments using immunoprecipitation with 20.6 [see Fig 4A (1) and 4B (2)] or B1G6 [see Fig4B (2) and an additional experiment (data not shown, 1)] were analyzed by densitometry scanning of PNGase F-treated samples. At each time point and for wild type CD1e (C) or $\Delta 12 \mathrm{CD} 1 \mathrm{e}(\Delta)$, the percentage of soluble among total immunoprecipitated CD1e $\alpha$ chains was determined. 
FIGURE 5. The $\Delta 12 \mathrm{CD} 1 \mathrm{e}$ form is immunologically active. A) Transfected M10 cells expressing CD1b alone $(\boldsymbol{\square})$ or co-expressing CD1b together with CD1e (O) or with $\triangle 12 \mathrm{CD} 1 \mathrm{e}(\square)$ were incubated with $\mathrm{PIM}_{6}(\mathrm{~A})$ or GM1 $(B)$ for $4 \mathrm{~h}$ before addition of specific $\mathrm{T}$ cell clones DL15A30 or GG33a, respectively. GM-CSF release was used as a read-out of T cell activation. $C$ ) In another type of experiment, M10 cells expressing CD1b alone were incubated with $20 \mu \mathrm{g} / \mathrm{ml} \mathrm{rsCD1e}(O)$ or rs $\Delta 12 \mathrm{CD} 1 \mathrm{e}(\square)$ or in the absence of rsCD1e ( $\mathbf{\square}$ ). After overnight incubation, cells were washed and incubated with $\mathrm{PIM}_{6}$ and specific $\mathrm{T}$ cells. IFN $\gamma$ release was used as a read-out of T cell response. D) PIM $_{6}$ [4] was digested with $\alpha$ mannosidase $(\alpha-\mathrm{m})$ in the presence of rsCD1e, rs $\Delta 12 \mathrm{CD} 1 \mathrm{e}$ or in their absence. The relative intensities of the peaks, corresponding to the different PIM species generated by digestion were measured by MALDI-MS mass spectrometry. 


\section{TABLE I}

Predicted N-terminal aminoacid sequences of human CD1 proteins and manmalian CD1e molecules after cleavage of their signal peptide

\begin{tabular}{|c|c|c|}
\hline & Proteins & Predicted N-terminal sequences ${ }^{a}$ \\
\hline \multirow{5}{*}{ A } & Human CD1a & DGLKEPLSFHVTWIASFYNHSWKQNLVSGW \\
\hline & Human CD1b & EHAFQGPTSFHVIQTSSFTNSTWAQTQGSGW \\
\hline & Human CD1C & DASQEHVSFHVIQIFSFVNQSWARGQGSGW \\
\hline & Human CD1d & EVPQRLFPLRCLQISSFANSSWTRTDGLAW \\
\hline & Human CD1e & APQALQSYHLAAEEQLSFRMLQTSSFANHSWAHSEGSGW \\
\hline \multirow{5}{*}{ B } & Human CD1e & APQALQSYHLAAEEQLS \\
\hline & Macaque CDle & APQALQSYRPAAEDPLS \\
\hline & Guinea-Pig CDle & GPQPLGWHHPAEAEEPLI \\
\hline & Pig CDle & ALQVLEPRHPAAEEPLS \\
\hline & Rabbit CD1e & GSEALRPHHPAAEEPLT \\
\hline
\end{tabular}

${ }^{a}$ The N-terminal sequences were predicted using http://www.cbs.dtu.dk/services/Signal/. The accession numbers of the aminoacid sequences of the human CD1a, b, c d and e proteins are NP_001754, NP_001755, CAG33361, AAA59673 and NP_112155, respectively. The accession numbers of the aminoacid sequences of macaque, pig, guinea-pig and rabbit CD1e are NP_001036123, NM_001034394, BAF38761, AAF12745 and AAG39380, respectively.

Table II

$\mathrm{N}$ - or C-terminal aminoacid sequences of human membrane-associated and soluble CD1e.

\begin{tabular}{|crrrl|}
\hline CDle form & m/z value $^{\mathrm{a}}$ & Mass $^{\mathrm{b}}$ & Sequence $^{\text {c }}$ & Loc $^{\mathrm{d}}$ \\
\hline mCDle & $720.30(3+)$ & 2158.08 & APQALQSYHLAAEEQLSFR & $\mathrm{N}$ \\
mCDle & $597.93(3+)$ & 1791.90 & LQSYHLAAEEQLSFR & $\mathrm{N}$ \\
mCDle & $650.79(3+)$ & 1299.66 & HLAAEEQLSFR & $\mathrm{N}$ \\
mCDle & $525.72(3+)$ & 1049.51 & AAEEQLSFR & $\mathrm{N}$ \\
\hline sCDle & $454.69(2+)$ & 907.44 & EEQLSFR & $\mathrm{N}$ \\
sCDle & $529.18(3+)$ & 1584.78 & HSSLFGGHDLIIHWGG & $\mathrm{C}$ \\
\hline
\end{tabular}

CD1e molecules were immunopurified from transfected cells and separated by SDS-PAGE (Fig. 2A). Tryptic fragments of the membrane-associated and soluble forms were analyzed by MALDI mass spectrometry (Fig 2B) and de novo sequencing after LC-MS/MS (Fig 2C).

${ }^{a} 2+$ and $3+$ indicate doubly-and triply- charged ions, respectively

b Monoisotopic molecular mass of the peptide

${ }^{\mathrm{c}}$ Sequences were determined by de novo sequencing after LC-MS/MS (Fig. 2C)

$\mathrm{d}$ Localization of the sequences on CD1e molecule. N, N-terminus, C, C-terminus. 
Figure 1

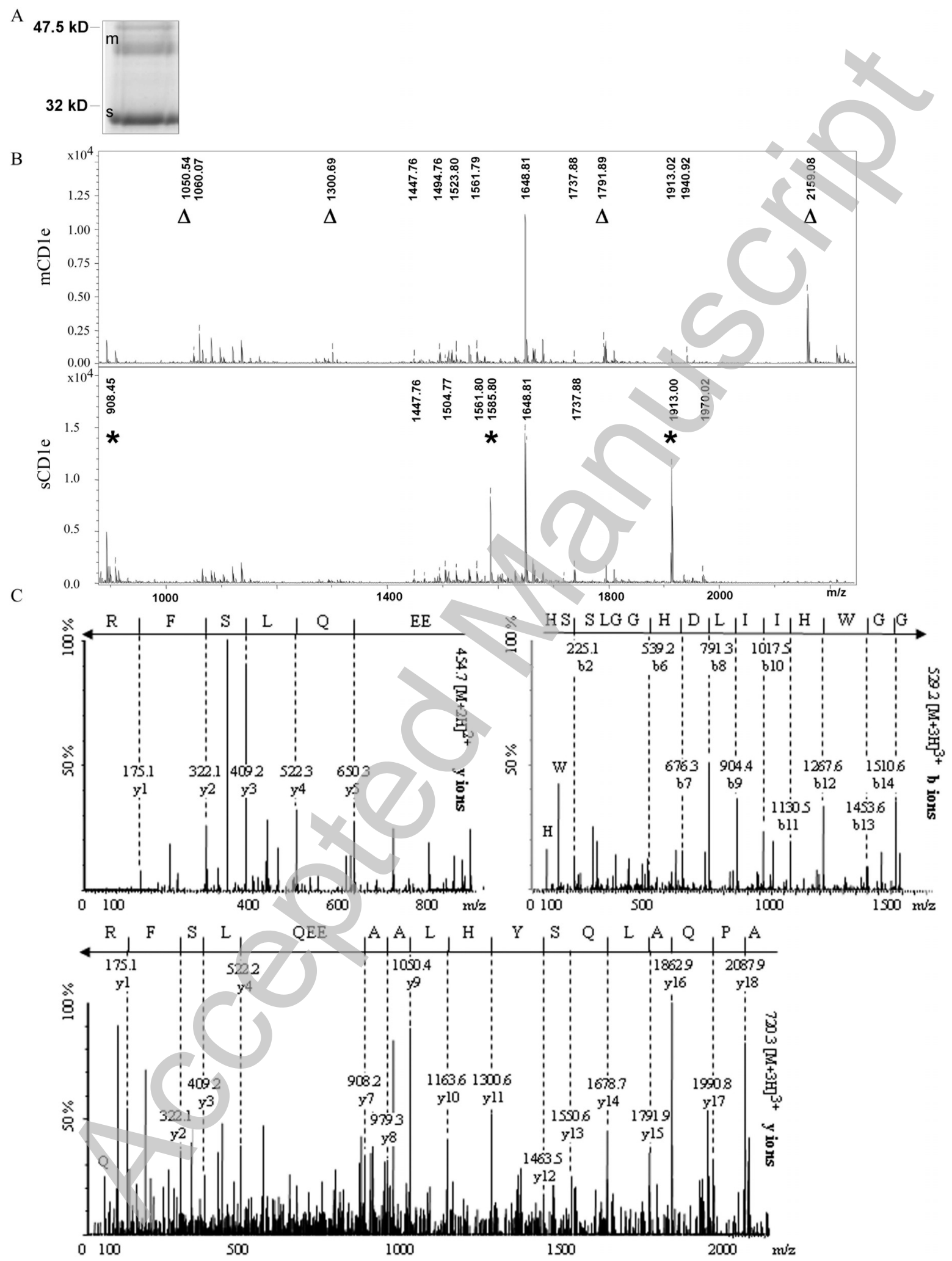


Figure 2

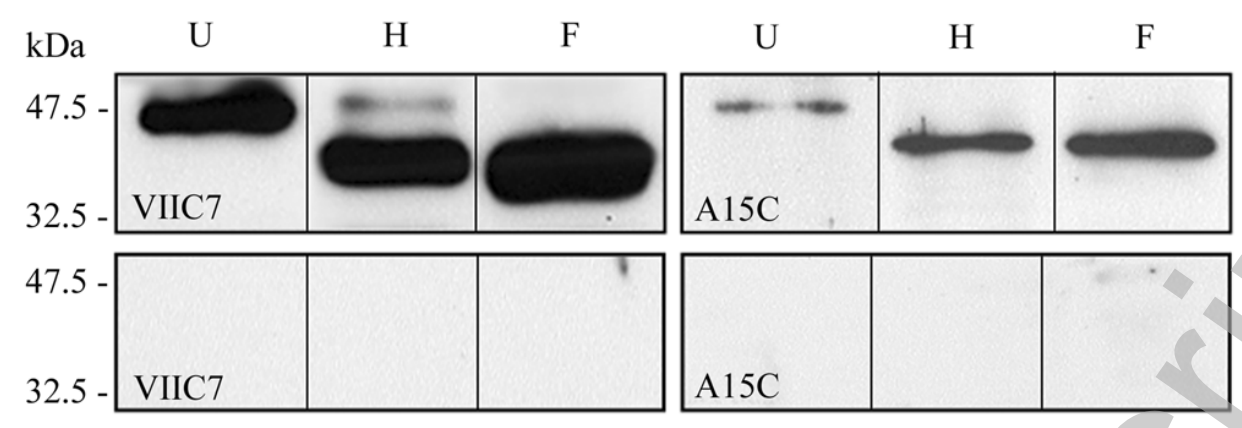

Figure 3

A

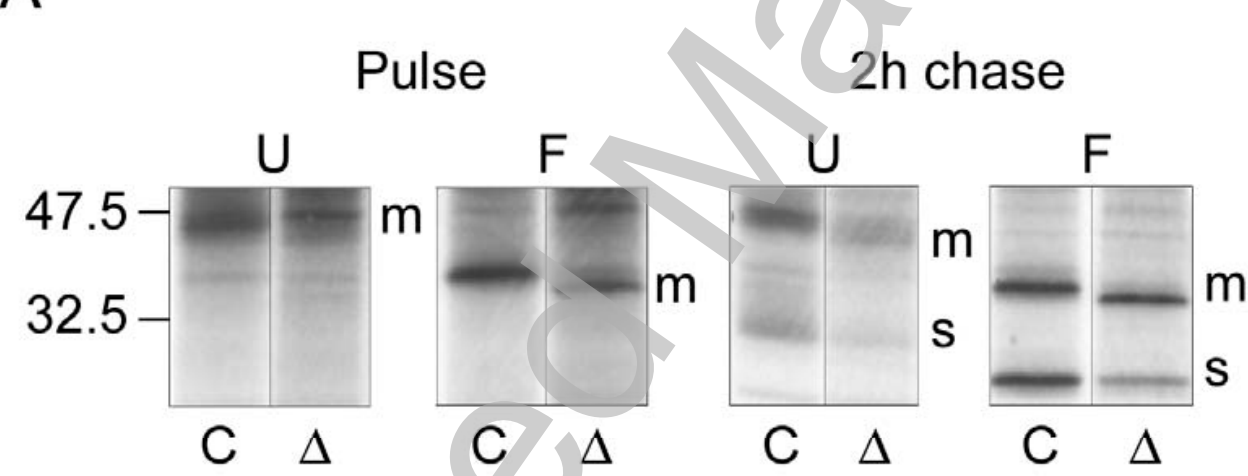

B

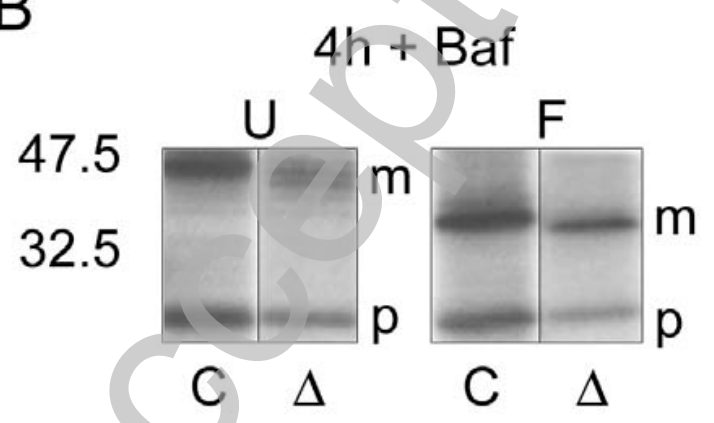




\section{Figure 4}
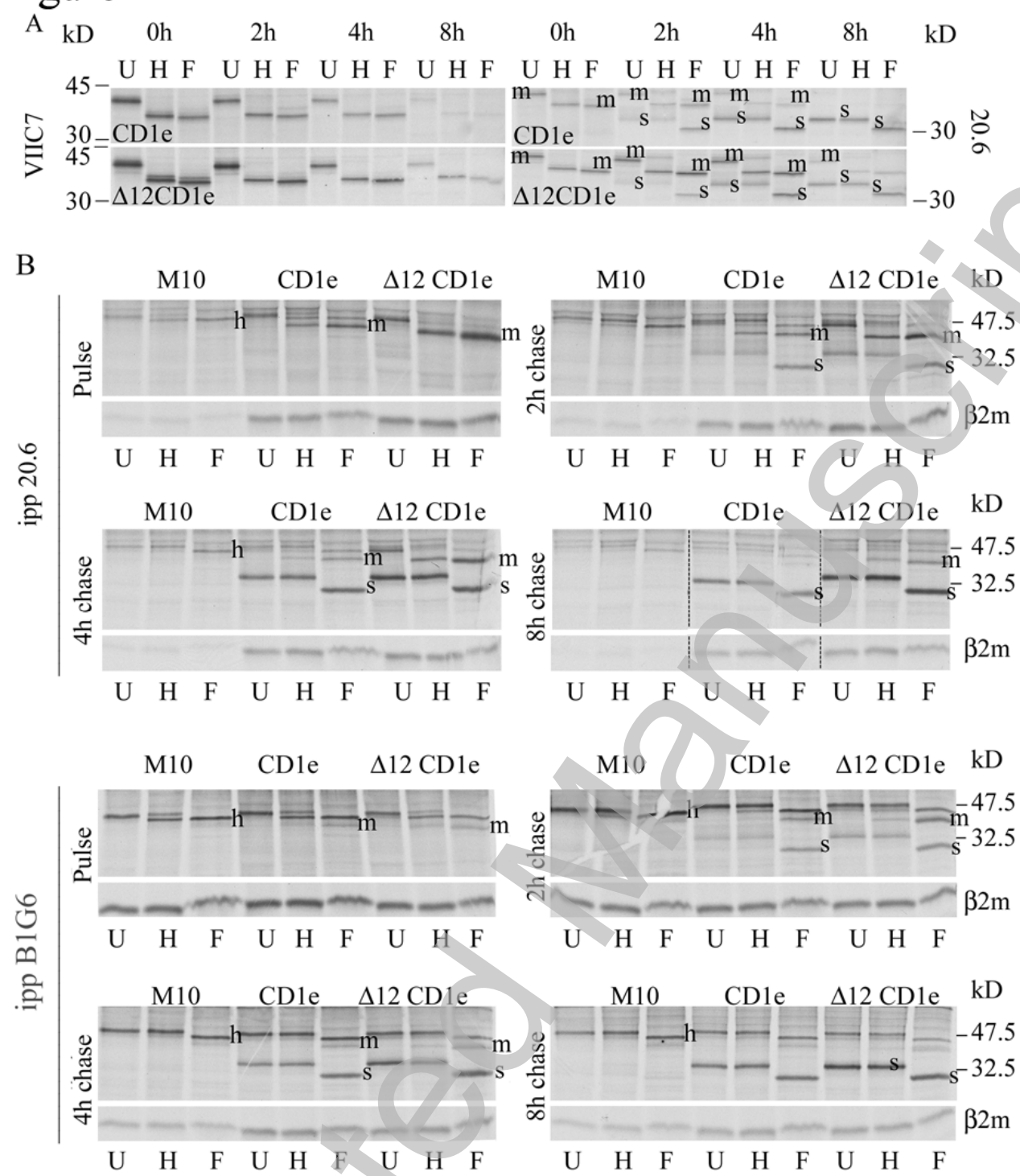


\section{Figure 4}

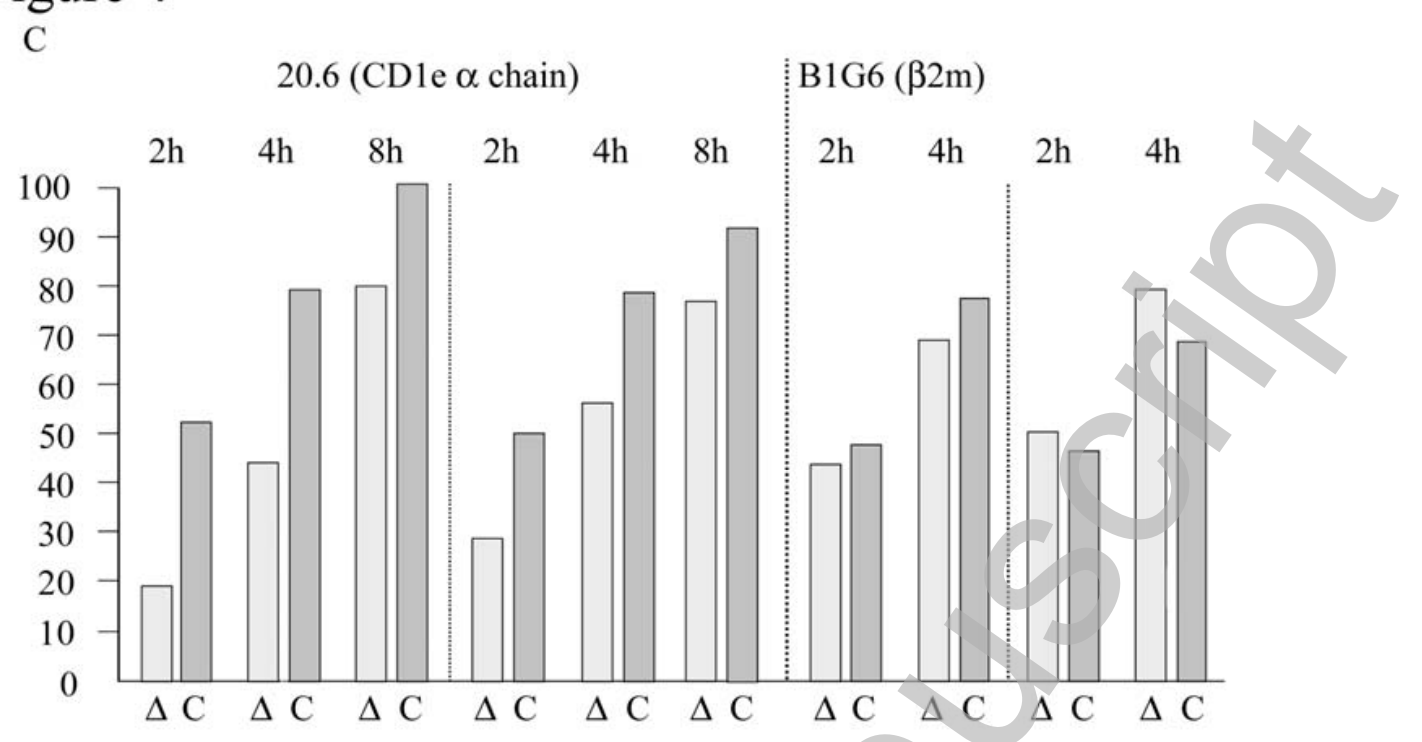




\section{Figure 5}
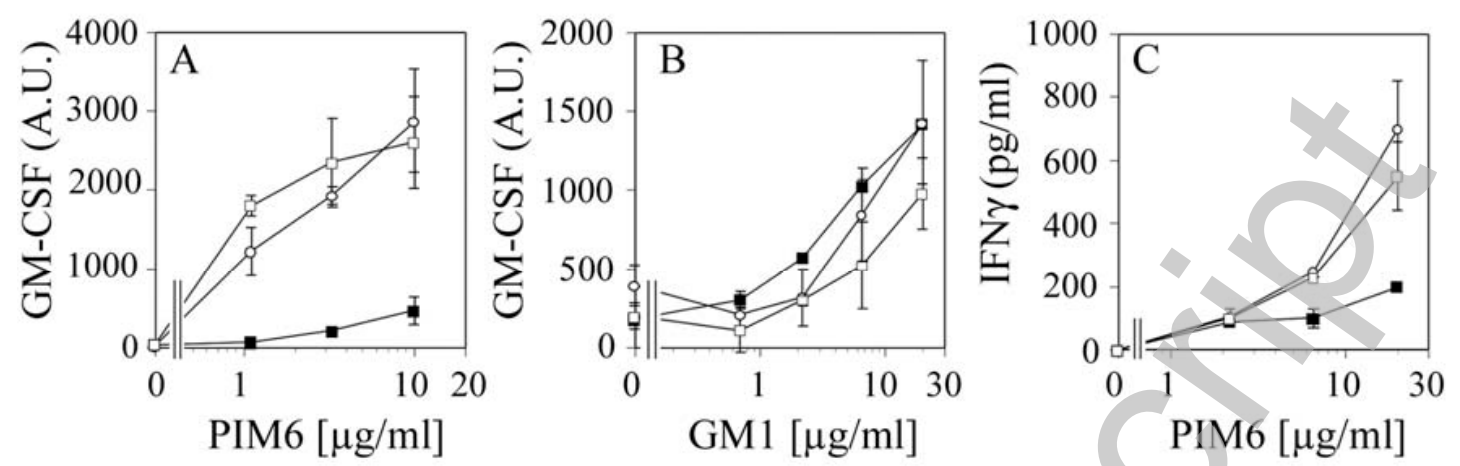

$\mathrm{D}$
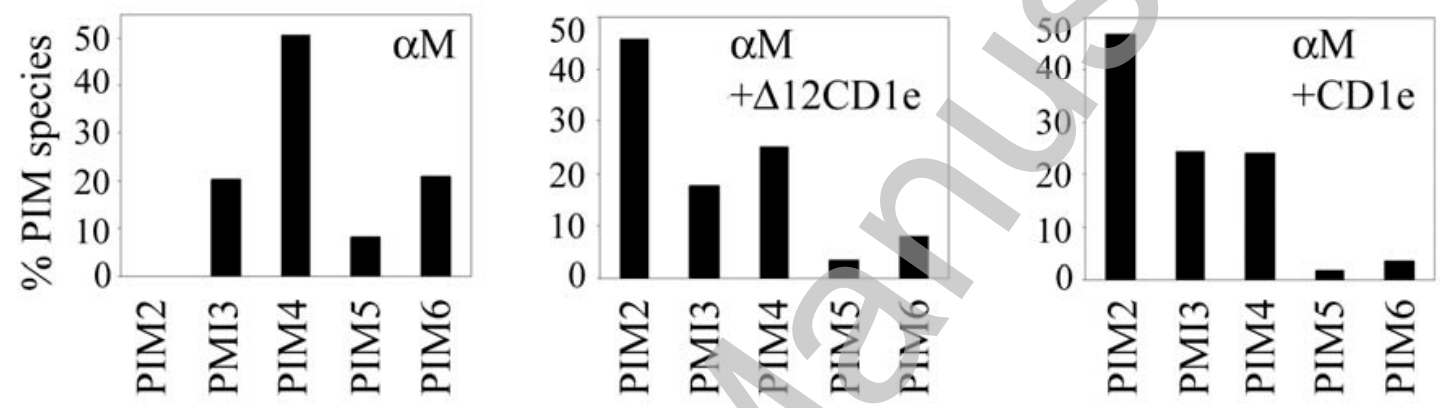